\title{
Hartree-Fock Simulation of Persistent Current in Rings with Single Scatterer
}

\author{
R. NÉmeth AND M. MošKO \\ Institute of Electrical Engineering, Slovak Academy of Sciences \\ Dúbravská cesta 9, 84104 Bratislava, Slovakia
}

\begin{abstract}
Using the self-consistent Hartree-Fock approximation for spinless electrons at zero temperature, we calculate the persistent current of the interacting electron gas in a one-dimensional ring containing a single $\delta$ barrier. Our results agree with correlated models like the Luttinger liquid model and lattice model with nearest-neighbor interaction. The persistent current is a sine-like function of magnetic flux. It decays with the ring length $(L)$ faster than $L^{-1}$ and eventually like $L^{-\alpha-1}$, where $\alpha>0$ is universal.
\end{abstract}

PACS numbers: 73.23.--b, 73.61.Ey

\section{Introduction}

Electrons in a quantum wire form a one-dimensional (1D) electron gas. By tying the wire ends to each other one forms a $1 \mathrm{D}$ ring. The ring is called "mesoscopic" if its circumference is shorter than the electron coherence length. Magnetic flux applied through the opening of such ring gives rise to an equilibrium persistent current [1]. Measurements of persistent currents are not yet fully understood due to the complicated role of the electron-electron (e-e) interaction and disorder [1]. Here we study the persistent current of interacting spinless electrons in a 1D ring with a single scatterer. Let us review this simplified problem.

For non-interacting electrons the persistent current $(I)$ as a function of the magnetic flux $(\phi)$ and ring circumference $(L)$ can be derived for an arbitrary scatterer [2]. For even number of electrons $(N)$ assumed in our paper, the result relation

$$
I=\frac{e v_{\mathrm{F}}}{\pi L} \frac{\arccos \left(\left|\tilde{t}_{k_{\mathrm{F}}}\right| \cos \left(\phi^{\prime}-\pi\right)\right)}{\sqrt{1-\left|\tilde{t}_{k_{\mathrm{F}}}\right|^{2} \cos ^{2}\left(\phi^{\prime}\right)}}\left|\tilde{t}_{k_{\mathrm{F}}}\right| \sin \left(\phi^{\prime}\right), \quad \phi^{\prime} \equiv 2 \pi \phi / \phi_{0},
$$

where $\phi_{0}=h / e$ is the flux quantum, $\tilde{t}_{k_{\mathrm{F}}}$ is the electron transmission amplitude through the bare scatterer, and $k_{\mathrm{F}}$ is the Fermi wave vector. For small $\tilde{t}_{k_{\mathrm{F}}}$ 


$$
I=\frac{e v_{\mathrm{F}}}{2 L}\left|\tilde{t}_{k_{\mathrm{F}}}\right| \sin \left(\phi^{\prime}\right)
$$

For a repulsive e-e interaction in the continuous ring, the spinless persistent current can still be derived analytically in the Luttinger liquid model [2]. For $L \rightarrow \infty$

$$
I \propto L^{-\alpha-1} \sin \left(\phi^{\prime}\right),
$$

where the power $\alpha>0$ is universal, depending only on the e-e interaction. Finally, the authors of Ref. [3] obtained the persistent current for interacting spinless electrons by solving the lattice model with nearest-neighbor hopping and interaction. Using the renormalization group (RG), they confirmed for a $1 \mathrm{D}$ ring with a single scatterer the existance of the current which decays faster than $1 / L$ and is a sine-like function of $\phi$. They found $I \propto L^{-\alpha-1} \sin \left(2 \pi \phi / \phi_{0}\right)$ for long chains and/or strong scatterers.

Here we find similar results within the Hartree-Fock model. Using the self-consistent Hartree-Fock approximation at zero temperature, we study the persistent current of the interacting spinless electron gas in a continuous 1D ring with a single $\delta$ barrier. Our results are in good accord with the Luttinger liquid model [2] and RG model [3]. We demonstrate $I \propto L^{-\alpha-1} \sin \left(2 \pi \phi / \phi_{0}\right)$ for strong scatterers.

\section{Theoretical model}

We consider $N$ interacting 1D electrons with free motion along a circular ring threaded by magnetic flux $\phi=B S=A L$, where $S$ is the area of the ring, $B$ is the magnetic field (constant and perpendicular to the ring area), and $A$ is the magnitude of the resulting vector potential (circulating along the ring circumference). In the Hartree-Fock approximation the many-body electron state is the Slater determinant of single-electron wave functions $\psi_{k}(x)$, where $k$ is the electron wave vector. These wave functions can be found by solving the Hartree-Fock equation

$$
\left[\frac{\hbar^{2}}{2 m}\left(-\mathrm{i} \frac{\partial}{\partial x}+\frac{2 \pi}{L} \frac{\phi}{\phi_{0}}\right)^{2}+\gamma \delta(x)+U_{\mathrm{H}}(x)+U_{\mathrm{F}}(k, x)\right] \psi_{k}(x)=\varepsilon_{k} \psi_{k}(x)
$$

with boundary condition $\psi_{k}(x+L)=\psi_{k}(x)$, where $m$ is the electron effective mass, $x$ is the electron coordinate along the ring, $\gamma \delta(x)$ is the potential of the scatterer,

$$
U_{\mathrm{H}}(x)=\sum_{k^{\prime}} \int \mathrm{d} x^{\prime} V\left(x-x^{\prime}\right)\left|\psi_{k^{\prime}}\left(x^{\prime}\right)\right|^{2}
$$

is the Hartree potential,

$$
U_{\mathrm{F}}(k, x)=-\frac{1}{\psi_{k}(x)} \sum_{k^{\prime}} \int \mathrm{d} x^{\prime} V\left(x-x^{\prime}\right) \psi_{k}\left(x^{\prime}\right) \psi_{k^{\prime}}^{*}\left(x^{\prime}\right) \psi_{k^{\prime}}(x)
$$


is the Fock nonlocal exchange potential, and $V\left(x-x^{\prime}\right)$ is the e-e interaction. In Eqs. (5) and (6) we sum over all occupied states $k^{\prime}$ up to the Fermi energy.

In the groundstate the persistent current is given by

$$
I=-\frac{\partial}{\partial \phi} E_{0}(\phi), \quad E_{0}=\sum_{k}\left[\varepsilon_{k}-\frac{1}{2}\left\langle\psi_{k}\left|U_{\mathrm{H}}(x)+U_{\mathrm{F}}(k, x)\right| \psi_{k}\right],\right.
$$

where $E_{0}$ is the eigenenergy of the groundstate (expressed in the Hartree-Fock approximation). Following Ref. [4], we simplify Eq. (6) as

$$
U_{\mathrm{F}}(x) \simeq-\sum_{k^{\prime}} \int \mathrm{d} x^{\prime} V\left(x-x^{\prime}\right) \operatorname{Re}\left[\psi_{k^{\prime}}^{*}\left(x^{\prime}\right) \psi_{k^{\prime}}(x)\right]
$$

by noticing that $\sum_{k^{\prime}} \psi_{k^{\prime}}^{*}\left(x^{\prime}\right) \psi_{k^{\prime}}(x) \simeq \delta\left(x-x^{\prime}\right)$. This "almost-closure relation" is a very good approximation, as shown in Ref. [4]. Applying the "almost-closure relation" and Eqs. (7) and (8) we obtain

$$
I=-\sum_{k}\left[\frac{\partial \varepsilon_{k}}{\partial \phi}-\left\langle\psi_{k}\left|\frac{\partial}{\partial \phi}\left(U_{\mathrm{H}}+U_{\mathrm{F}}\right)\right| \psi_{k}\right\rangle\right] .
$$

Approximation (8) saves computational time and allows us to study long rings.

We solve Eq. (4) self-consistently by means of the method described in detail elsewhere [5]. The output of the method are the single-particle states $\psi_{k}(x)$ and $\varepsilon_{k}$, the Hartree-Fock potential $U_{\mathrm{H}}(x)+U_{\mathrm{F}}(x)$, and eventually the persistent current (9). Below we present numerical results for the GaAs ring with electron density $n=5 \times 10^{7} \mathrm{~m}^{-1}$, effective mass $m=0.067 m_{0}$, and e-e interaction

$$
V\left(x-x^{\prime}\right)=V_{0} \exp \left(-\left|x-x^{\prime}\right| / d\right),
$$

where $V_{0}=34 \mathrm{meV}$ and $d=3 \mathrm{~nm}$. We adopt the finite-ranged interaction (10) because we want to compare it with the correlated models which also rely on the e-e interaction of finite range. Interaction (10) is a reasonably chosen screened interaction, used to study the many-body $1 \mathrm{D}$ models [6].

\section{Results}

The transmission and reflection amplitudes of the $\delta$ barrier are $\tilde{t}_{k}=k /(k+\mathrm{i} \zeta)$ and $\tilde{r}_{k}=-\mathrm{i} \zeta /(k+\mathrm{i} \zeta)$, where $\zeta=\gamma m / \hbar^{2}$. Since $k_{\mathrm{F}}$ and $m$ are fixed, instead of using $\gamma$ we parametrize the $\delta$ barrier by its transmission coefficient $\left|\tilde{t}_{k_{\mathrm{F}}}\right|^{2}$.

In panel $a$ of Fig. 1 we show the persistent current $L I\left(\phi^{\prime}\right)$ calculated by our self-consistent Hartree-Fock approach, in panel $b$ we show the RG result of Ref. [3] in terms of $N I\left(\phi^{\prime}\right)$. All $L I$ data are normalized by $e v_{\mathrm{F}} / 2$. The ring of Ref. [3] is a tight-binding lattice at half-filling, so we cannot expect quantitative agreement. We see excellent qualitative agreement. In both cases the e-e interaction preserves the sine-like dependence on $\phi^{\prime}$ and the current decays faster than $1 / L$. The authors of Ref. [3] also performed the (non-self-consistent) Hartree-Fock calculation. The calculation is shown in panel $c$. It fails to fit the sine-like shape. 

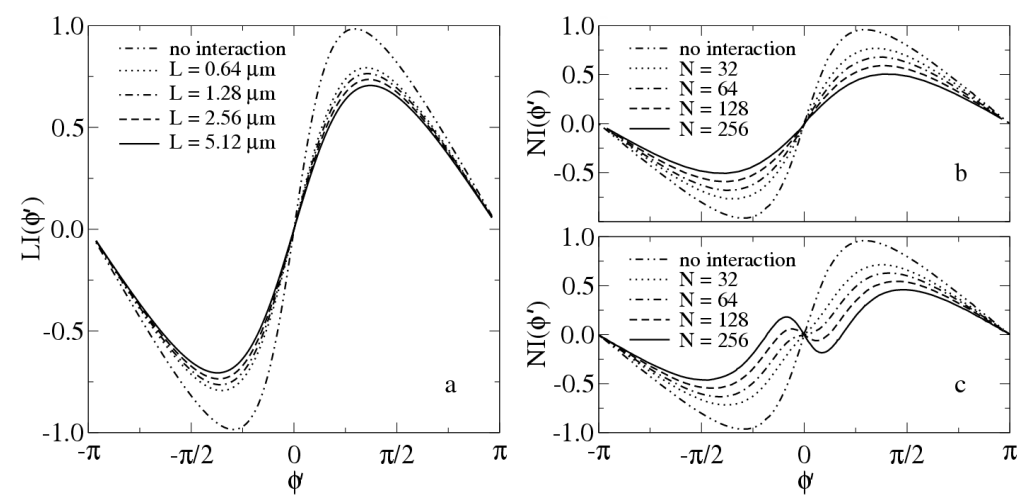

Fig. 1. Panel $a$ shows the persistent current $L I(\phi)$, calculated by our self-consistent Hartree-Fock approach for $\left|\tilde{t}_{k_{\mathrm{F}}}\right|^{2}=0.64$ and various $L$. For qualitative comparison, panels $b$ and $c$ show the data from Fig. 3 of Ref. [3]. These data were obtained [3] for a ring-shaped lattice model with the same $\left|\tilde{t}_{k_{\mathrm{F}}}\right|^{2}$ and same $N$ as in panel $a$, where $N=32,64, \ldots$ for $L=0.64 \mu \mathrm{m}, 1.28 \mu \mathrm{m}, \ldots$ Panel $b$ shows the results of the RG solution with correlations included. Panel $c$ shows the results of the (non-self-consistent) Hartree-Fock solution.

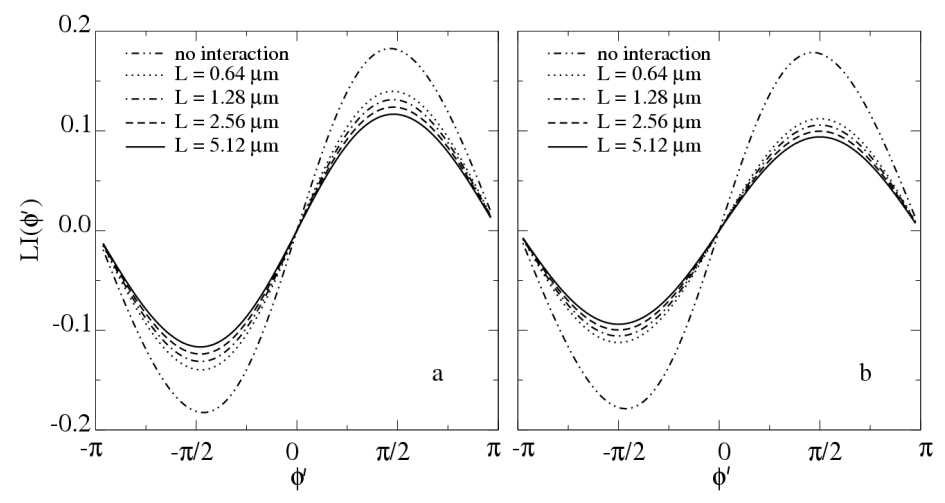

Fig. 2. Persistent current $L I\left(\phi^{\prime}\right)$ for $\left|\tilde{t}_{k_{\mathrm{F}}}\right|^{2}=0.03$ and various $L$. Panel $a$ shows our Hartree-Fock data, panel $b$ the Luttinger liquid scaling (see the text).

In Fig. 2 we present the persistent current $L I\left(\phi^{\prime}\right)$ for the $\delta$ barrier as strong as $\left|\tilde{t}_{k_{\mathrm{F}}}\right|^{2}=0.03$. Our self-consistent Hartree-Fock results are shown in panel $a$. We want to compare these results with the scaling law due to the Luttinger liquid model (Eq. (3)). To evaluate the scaling law (3), we reformulate it as follows [2]. We replace the bare transmission amplitude $\tilde{t}_{k_{\mathrm{F}}}$ in the non-interacting scaling law (2) by the transmission amplitude of the interacting electron gas [7],

$$
t_{k_{\mathrm{F}}}=\frac{\tilde{t}_{k_{\mathrm{F}}}(d / L)^{\alpha}}{\sqrt{\left|\tilde{r}_{k_{\mathrm{F}}}\right|^{2}+\left|\tilde{t}_{k_{\mathrm{F}}}\right|^{2}(d / L)^{2 \alpha}}} \simeq \frac{\tilde{t}_{k_{\mathrm{F}}}}{\left|\tilde{r}_{k_{\mathrm{F}}}\right|}(d / L)^{\alpha},
$$


where $d$ is the range of the e-e interaction $V\left(x-x^{\prime}\right)$ and the right hand side of (11) holds for small $\tilde{t}_{k_{\mathrm{F}}}$ and/or large $L$. For small $\tilde{t}_{k_{\mathrm{F}}}$ one indeed obtains the scaling

$$
I=\omega L^{-\alpha-1} \sin \left(\phi^{\prime}\right),
$$

where $\omega=e v_{\mathrm{F}}\left|\tilde{t}_{k_{\mathrm{F}}}\right| d^{\alpha} / 2\left|\tilde{r}_{k_{\mathrm{F}}}\right|$. The formula (11) was derived [7] by the RG method in the limit of weak e-e interaction $(\alpha \ll 1)$. In this limit $\alpha$ is given by [7]

$$
\alpha=\frac{V(0)-V\left(2 k_{\mathrm{F}}\right)}{2 \pi \hbar v_{\mathrm{F}}},
$$

where $V(q)$ is the Fourier transform of the e-e interaction $V\left(x-x^{\prime}\right)$. The Fourier transform of our interaction (10) reads $V(q)=2 V_{0} d /\left(1+q^{2} d^{2}\right)$. We set this formula and our parameters into Eq. (13). We obtain $\alpha=0.0855$. We evaluate the formula (12) in panel $b$ of Fig. 2. Clearly, this formula and the Hartree-Fock result of panel $a$ are in excellent qualitative and good quantitative agreement.

The left panel of Fig. 3 shows the persistent current $L I\left(\phi^{\prime}=\pi / 2\right)$ as a function of $L$ for various $\delta$ barriers. Our Hartree-Fock results are shown by open symbols connected by dashed lines. The full lines in the left panel show the scaling
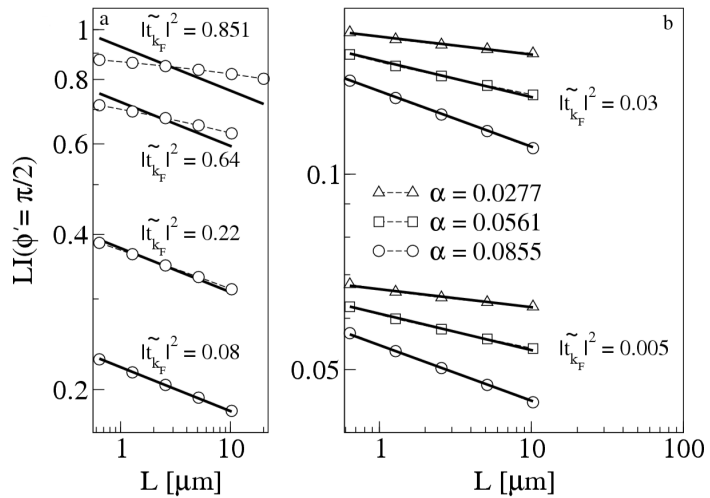

Fig. 3. Persistent current $L I\left(\phi^{\prime}=\pi / 2\right)$ as a function of $L$ for various $\delta$ barriers (panel $a$ ) and various e-e interaction strengths $\alpha$ (panel $b$ ). Our Hartree-Fock results are shown by open symbols connected by dashed lines. The full lines show the scaling law (12) with the power $\alpha$ given by Eq. (13) and with the prefactor $\omega$ adjusted to fit the HartreeFock data quantitatively. The powers $\alpha=0.0277,0.0561$, and 0.0855 correspond to $V_{0}=11 \mathrm{meV}, 22.3 \mathrm{meV}$, and $34 \mathrm{meV}$, respectively, with $d$ fixed to $3 \mathrm{~nm}$. If we adjust the same $\alpha$ for some other choice of $V_{0}$ and $d$, our results remain unchanged.

law (12) with the power $\alpha=0.0855$ obtained from the universal formula (13) and with the factor $\omega$ adjusted to fit the open symbols quantitatively. We see that the Hartree-Fock data reproduce the universal law $I \propto L^{-\alpha-1}$ in the limit of small $\tilde{t}_{k_{\mathrm{F}}}$. The power law (12) is asymptotic and does not work for large $\tilde{t}_{k_{\mathrm{F}}}$. Eventually, for small enough $\tilde{t}_{k_{\mathrm{F}}}$ our Hartree-Fock data always follow the scaling law $I \propto L^{-\alpha-1}$. This is demonstrated in the right panel for various $\alpha$ and small $\tilde{t}_{k_{\mathrm{F}}}$. 
Finally, we discuss the Hartree-Fock potential $U_{\mathrm{H}}(x)+U_{\mathrm{F}}(x)$ normalized as

$$
U_{\mathrm{HF}}(y)=(d / L)^{\alpha}\left[U_{\mathrm{H}}(y)+U_{\mathrm{F}}(y)\right] / \Delta_{L}, \quad y=x / L, \quad \Delta_{L}=\pi \hbar v_{\mathrm{F}} / L .
$$

In absence of the $\delta$ barrier, the potentials $U_{\mathrm{H}}(x)$ and $U_{\mathrm{F}}(x)$ are $x$-independent constants which we set to zero as they only imply a constant energy shift. The $\delta$ barrier induces the Friedel oscillations of $U_{\mathrm{H}}(x)$ and $U_{\mathrm{F}}(x)$ around this zero mean.

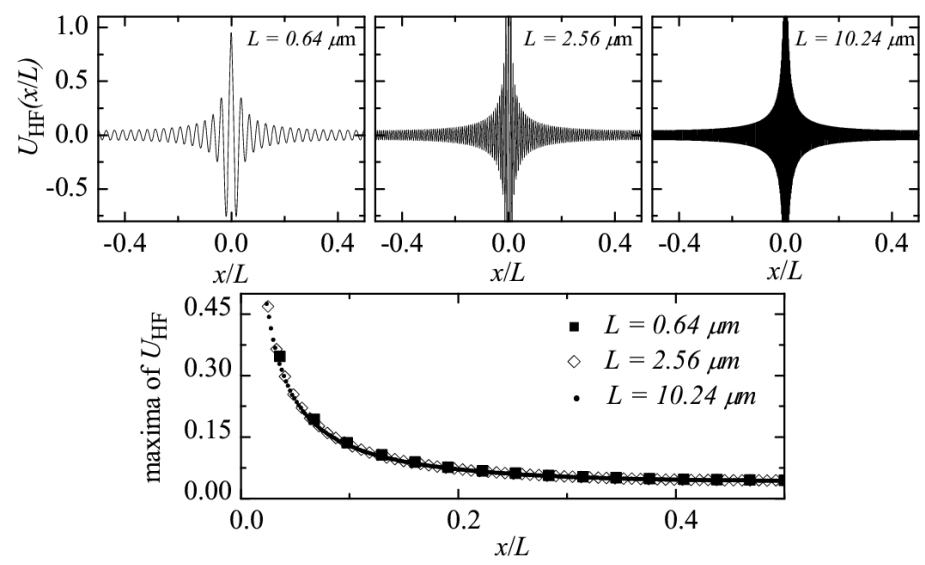

Fig. 4. Self-consistent Hartree-Fock potential $U_{\mathrm{HF}}(x / L)$ along a $1 \mathrm{D}$ ring, induced by the (not shown) $\delta$ barrier at $x=0$. The $\delta$ barrier is adjusted to have the transmission $\left|\tilde{t}_{k_{\mathrm{F}}}\right|^{2}=0.005$ at the Fermi level $(14 \mathrm{meV})$. The ring is threaded by magnetic flux $\phi=0.25 \phi_{0}$, the ring length, $L$, is varied as a parameter. The bottom panel shows just the maxima of the Friedel oscillations.

In Fig. 4 we show the typical self-consistent $U_{\mathrm{HF}}(y)$ in the ring with a strong scatterer at $y=0$. The potential exhibits Friedel oscillations with period $\lambda_{\mathrm{F}} / 2 L$. The bare scatterer is thus "dressed" by an extra scatterer due to the Friedel oscillations. This is why we see the persistent current to decay faster than $1 / L$. It is more difficult to understand why we see just $L I \propto(d / L)^{\alpha}$. As $L$ increases, the Friedel oscillations in Fig. 4 are too dense to be distinguishable, but we can observe asymptotic decay of their amplitudes. Notice that the "envelope" of the oscillation amplitude of $U_{\mathrm{HF}}(y)$ is the same for all $L$. Indeed, as shown in the bottom panel, maxima of the Friedel oscillations scale for all $L$ to a single curve. Notice also (c.f. Eq. (14) that $U_{\mathrm{HF}}(y)$ involves the scaling factor $(d / L)^{\alpha}$. This might be the reason why $L I \propto(d / L)^{\alpha}$, but we have so far not found a clear interpretation.

\section{Conclusions}

In conclusion, using the self-consistent Hartree-Fock approximation at zero temperature, we have calculated the persistent current of the weakly-interacting spinless electron gas in a $1 \mathrm{D}$ ring with a single $\delta$ barrier. Our results systematically 
agree with correlated models like the Luttinger liquid model [2] and RG model [3]. For strong $\delta$ barriers we reproduce the scaling law $I \propto L^{-\alpha-1} \sin \left(2 \pi \phi / \phi_{0}\right)$. These non-trivial findings show that the self-consistent Hartree-Fock approximation is reliable in this context, and the approximation (8) as well.

\section{Acknowledgments}

We thank for the APVT grant APVT-51-021602 and VEGA grant $2 / 3118 / 23$.

\section{References}

[1] Y. Imry, Introduction to Mesoscopic Physics, Oxford University Press, Oxford (UK) 2002.

[2] A.O. Gogolin, N.V. Prokof'ev, Phys. Rev. B 50, 4921 (1994).

[3] V. Meden, U. Schollwöck, Phys. Rev. B 67, 035106 (2003).

[4] A. Cohen, R. Berkovits, A. Heinrich, Int. J. Mod. Phys. B 11, 1845 (1997).

[5] R. Nemeth, M. Mosko, cond-mat/0503146.

[6] V. Meden, W. Metzner, U. Schollwöck, O. Schneider, T. Stauber, K. Schönhammer, Europhys. J. B 16, 631 (2000).

[7] K.A. Matveev, D. Yue, L.I. Glazman, Phys. Rev. Lett. 71, 3351 (1993). 